\title{
Fixed point results of pointwise contractions in modular metric spaces
}

\author{
Afrah A N Abdou ${ }^{1 *}$ and Mohamed A Khamsi ${ }^{2,3}$
}

"Correspondence:

aabdou@kau.edu.sa

${ }^{1}$ Department of Mathematics, King Abdulaziz University, P.O. Box 80203

Jeddah, 21589, Saudi Arabia

Full list of author information is

available at the end of the article

\begin{abstract}
The notion of a modular metric on an arbitrary set and the corresponding modular spaces, generalizing classical modulars over linear spaces like Orlicz spaces, were recently introduced. In this paper we investigate the existence of fixed points of modular contractive mappings in modular metric spaces. These are related to the successive approximations of fixed points (via orbits) which converge to the fixed points in the modular sense, which is weaker than the metric convergence.
\end{abstract}

MSC: Primary 47H09; secondary 46B20; 47H10; 47E10

Keywords: $\Delta_{2}$-condition; fixed point; modular function space; modular metric spaces; Orlicz spaces; pointwise contraction mapping

\section{Introduction}

The purpose of this paper is to give an outline of a fixed point theory for Lipschitzian mappings defined on some subsets of modular metric spaces which are natural generalizations of both function and sequence variants of many important, from applications perspective, spaces like Lebesgue, Orlicz, Musielak-Orlicz, Lorentz, Orlicz-Lorentz, CalderonLozanovskii spaces and many others. Modular metric spaces were introduced in [1, 2]. The main idea behind this new concept is the physical interpretation of the modular. Informally speaking, whereas a metric on a set represents nonnegative finite distances between any two points of the set, a modular on a set attributes a nonnegative (possibly, infinite valued) 'field of (generalized) velocities': to each 'time' $\lambda>0$ (the absolute value of) an average velocity $w_{\lambda}(x, y)$ is associated in such a way that in order to cover the - 'distance' between points $x, y \in X$, it takes time $\lambda$ to move from $\mathrm{x}$ to $\mathrm{y}$ with velocity $w_{\lambda}(x, y)$. But the way we approached the concept of modular metric spaces is different. Indeed, we look at these spaces as the nonlinear version of the classical modular spaces as introduced by Nakano [3] on vector spaces and modular function spaces introduced by Musielack [4] and Orlicz [5].

In recent years, there was an uptake of interest in the study of electrorheological fluids, sometimes referred to as 'smart fluids' (for instance, lithium polymetachrylate). For these fluids, modeling with sufficient accuracy using classical Lebesgue and Sobolev spaces, $L^{p}$ and $W^{1, p}$, where $p$ is a fixed constant, is not adequate, but rather the exponent $p$ should be able to vary $[6,7]$. One of the most interesting problems in this setting is the famous Dirichlet energy problem $[8,9]$. The classical technique used so far in studying this problem is to convert the energy functional, naturally defined by a modular, to a convoluted

(c) 2013 Abdou and Khamsi; licensee Springer. This is an Open Access article distributed under the terms of the Creative Commons Attribution License (http://creativecommons.org/licenses/by/2.0), which permits unrestricted use, distribution, and reproduction in any medium, provided the original work is properly cited. 
and complicated problem which involves a norm (the Luxemburg norm). The modular metric approach is more natural and has not been used extensively.

The fixed point property in modular function spaces was initiated after the publication of the paper [10] in 1990. More recently, the authors presented a series of fixed point results for pointwise contractions and asymptotic pointwise contractions acting in modular functions spaces [11,12]. In this paper, we define and investigate the fixed point property in the framework of modular metric spaces. It seems that a timid start has been going on for a few years now but it still lacks some generalities. The importance of applications of fixed points of mappings in modular metric spaces follows the success of such applications in modular function spaces because of the richness of structure of modular function spaces that - besides being Banach spaces (or F-spaces in a more general settings) - are equipped with modular equivalents of norm or metric notions, and also are equipped with almost everywhere convergence and convergence in submeasure. In many cases, particularly in applications to integral operators, approximation and fixed point results, modular type conditions are much more natural as modular-type assumptions can be more easily verified than their metric or norm counterparts. From this perspective, the fixed point theory in modular metric spaces should be considered as complementary to the fixed point theory in modular function spaces and in metric spaces. The theory of contractions and nonexpansive mappings defined on convex subsets of Banach spaces has been well developed since the 1960s (see, e.g., Belluce and Kirk [13, 14], Browder [15], Bruck [16], DeMarr [17], and Lim [18]) and generalized to other metric spaces (see, e.g., [19-21]) and modular function spaces (see, e.g., [10]). The corresponding fixed point results were then extended to larger classes of mappings like pointwise contractions [22,23] and asymptotic pointwise contractions and nonexpansive mappings [24, 25].

For more on metric fixed point theory, the reader may consult the book [26] and for modular metric spaces [27].

\section{Basic definitions and properties}

Let $\mathrm{X}$ be a nonempty set. Throughout this paper, for a function $w:(0, \infty) \times X \times X \rightarrow$ $(0, \infty)$, we write

$$
w_{\lambda}(x, y)=w(\lambda, x, y)
$$

for all $\lambda>0$ and $x, y \in X$.

Definition 2.1 $[1,2]$ A function $w:(0, \infty) \times X \times X \rightarrow[0, \infty]$ is said to be modular metric on $\mathrm{X}$ if it satisfies the following axioms:

(i) $x=y$ if and only if $w_{\lambda}(x, y)=0$ for all $\lambda>0$;

(ii) $w_{\lambda}(x, y)=w_{\lambda}(y, x)$ for all $\lambda>0$ and $x, y \in X$;

(iii) $w_{\lambda+\mu}(x, y) \leq w_{\lambda}(x, z)+w_{\mu}(z, y)$ for all $\lambda, \mu>0$ and $x, y, z \in X$.

If instead of (i) we have only the condition (i')

$$
w_{\lambda}(x, x)=0 \quad \text { for all } \lambda>0, x \in X \text {, }
$$


then $w$ is said to be a pseudomodular (metric) on $X$. A modular metric $w$ on $X$ is said to be regular if the following weaker version of (i) is satisfied:

$$
x=y \quad \text { if and only if } \quad w_{\lambda}(x, y)=0 \quad \text { for some } \lambda>0 .
$$

Finally, $w$ is said to be convex if, for $\lambda, \mu>0$ and $x, y, z \in X$, it satisfies the inequality

$$
w_{\lambda+\mu}(x, y) \leq \frac{\lambda}{\lambda+\mu} w_{\lambda}(x, z)+\frac{\mu}{\lambda+\mu} w_{\mu}(z, y)
$$

Note that for a metric pseudomodular $w$ on a set $X$, and any $x, y \in X$, the function $\lambda \rightarrow$ $w_{\lambda}(x, y)$ is nonincreasing on $(0, \infty)$. Indeed, if $0<\mu<\lambda$, then

$$
w_{\lambda}(x, y) \leq w_{\lambda-\mu}(x, x)+w_{\mu}(x, y)=w_{\mu}(x, y) .
$$

Definition 2.2 $[1,2]$ Let $w$ be a pseudomodular on $X$. Fix $x_{0} \in X$. The two sets

$$
X_{w}=X_{w}\left(x_{0}\right)=\left\{x \in X: w_{\lambda}\left(x, x_{0}\right) \rightarrow 0 \text { as } \lambda \rightarrow \infty\right\}
$$

and

$$
X_{w}^{*}=X_{w}^{*}\left(x_{0}\right)=\left\{x \in X: \exists \lambda=\lambda(x)>0 \text { such that } w_{\lambda}\left(x, x_{0}\right)<\infty\right\}
$$

are said to be modular spaces (around $x_{0}$ ).

It is clear that $X_{w} \subset X_{w}^{*}$, but this inclusion may be proper in general. It follows from [1,2] that if $w$ is a modular on $X$, then the modular space $X_{w}$ can be equipped with a (nontrivial) metric, generated by $w$ and given by

$$
d_{w}(x, y)=\inf \left\{\lambda>0: w_{\lambda}(x, y) \leq \lambda\right\}
$$

for any $x, y \in X_{w}$. If $w$ is a convex modular on $X$, according to [1,2], the two modular spaces coincide, $X_{w}^{*}=X_{w}$, and this common set can be endowed with the metric $d_{w}^{*}$ given by

$$
d_{w}^{*}(x, y)=\inf \left\{\lambda>0: w_{\lambda}(x, y) \leq 1\right\}
$$

for any $x, y \in X_{w}$. These distances will be called Luxemburg distances (see example below for the justification).

Next we give the main example that motivated this paper.

Example 2.1 Let $\Omega$ be a nonempty set and $\Sigma$ be a nontrivial $\sigma$-algebra of subsets of $\Omega$. Let $\mathcal{P}$ be a $\delta$-ring of subsets of $\Omega$ such that $E \cap A \in \mathcal{P}$ for any $E \in \mathcal{P}$ and $A \in \Sigma$. Let us assume that there exists an increasing sequence of sets $K_{n} \in \mathcal{P}$ such that $\Omega=\cup K_{n}$. By $\mathcal{E}$ we denote the linear space of all simple functions with supports from $\mathcal{P}$. By $\mathcal{M}_{\infty}$ we denote the space of all extended measurable functions, i.e., all functions $f: \Omega \rightarrow[-\infty, \infty]$ such that there exists a sequence $\left\{g_{n}\right\} \subset \mathcal{E},\left|g_{n}\right| \leq|f|$ and $g_{n}(\omega) \rightarrow f(\omega)$ for all $\omega \in \Omega$. By $1_{A}$ we denote the characteristic function of the set $A$. Let $\rho: \mathcal{M}_{\infty} \rightarrow[0, \infty]$ be a nontrivial, convex and even function. We say that $\rho$ is a regular convex function pseudomodular if: 
(i) $\rho(0)=0$;

(ii) $\rho$ is monotone, i.e., $|f(\omega)| \leq|g(\omega)|$ for all $\omega \in \Omega$ implies $\rho(f) \leq \rho(g)$, where $f, g \in \mathcal{M}_{\infty}$

(iii) $\rho$ is orthogonally subadditive, i.e., $\rho\left(f 1_{A \cup B}\right) \leq \rho\left(f 1_{A}\right)+\rho\left(f 1_{B}\right)$ for any $A, B \in \Sigma$ such that $A \cap B \neq \emptyset, f \in \mathcal{M}$;

(iv) $\rho$ has the Fatou property, i.e., $\left|f_{n}(\omega)\right| \uparrow|f(\omega)|$ for all $\omega \in \Omega$ implies $\rho\left(f_{n}\right) \uparrow \rho(f)$, where $f \in \mathcal{M}_{\infty}$;

(v) $\rho$ is order continuous in $\mathcal{E}$, i.e., $g_{n} \in \mathcal{E}$ and $\left|g_{n}(\omega)\right| \downarrow 0$ implies $\rho\left(g_{n}\right) \downarrow 0$.

Similarly, as in the case of measure spaces, we say that a set $A \in \Sigma$ is $\rho$-null if $\rho\left(g 1_{A}\right)=0$ for every $g \in \mathcal{E}$. We say that a property holds $\rho$-almost everywhere if the exceptional set is $\rho$-null. As usual, we identify any pair of measurable sets whose symmetric difference is $\rho$-null as well as any pair of measurable functions differing only on a $\rho$-null set. With this in mind, we define

$$
\mathcal{M}(\Omega, \Sigma, \mathcal{P}, \rho)=\left\{f \in \mathcal{M}_{\infty} ;|f(\omega)|<\infty \rho \text {-a.e. }\right\}
$$

where each $f \in \mathcal{M}(\Omega, \Sigma, \mathcal{P}, \rho)$ is actually an equivalence class of functions equal $\rho$-a.e. rather than an individual function. Where no confusion exists, we write $\mathcal{M}$ instead of $\mathcal{M}(\Omega, \Sigma, \mathcal{P}, \rho)$. Let $\rho$ be a regular function pseudomodular.

(a) We say that $\rho$ is a regular function semimodular if $\rho(\alpha f)=0$ for every $\alpha>0$ implies $f=0 \rho$-a.e.;

(b) We say that $\rho$ is a regular function modular if $\rho(f)=0$ implies $f=0 \rho$-a.e.

The class of all nonzero regular convex function modulars defined on $\Omega$ is denoted by $\Re$. Let us denote $\rho(f, E)=\rho\left(f 1_{E}\right)$ for $f \in \mathcal{M}, E \in \Sigma$. It is easy to prove that $\rho(f, E)$ is a function pseudomodular in the sense of Def. 2.1.1 in [27] (more precisely, it is a function pseudomodular with the Fatou property). Therefore, we can use all results of the standard theory of modular function space as per the framework defined by Kozlowski in [27-29]; see also Musielak [4] for the basics of the general modular theory. Let $\rho$ be a convex function modular.

(a) The associated modular function space is the vector space $L_{\rho}(\Omega, \Sigma)$, or briefly $L_{\rho}$, defined by

$$
L_{\rho}=\{f \in \mathcal{M} ; \rho(\lambda f) \rightarrow 0 \text { as } \lambda \rightarrow 0\} .
$$

(b) The following formula defines a norm in $L_{\rho}$ (frequently called the Luxemburg norm):

$$
\|f\|_{\rho}=\inf \{\alpha>0 ; \rho(f / \alpha) \leq 1\}
$$

A modular function space furnishes a wonderful example of a modular metric space. Indeed, let $L_{\rho}$ be a modular function space. Define the function $w$ by

$$
w_{\lambda}(f, g)=\rho\left(\frac{f-g}{\lambda}\right)
$$


for all $\lambda>0$ and $f, g \in L_{\rho}$. Then $w$ is a modular metric on $L_{\rho}$. Note that $w$ is convex if and only if $\rho$ is convex. Moreover, we have

$$
\|f-g\|_{\rho}=d_{w}^{*}(f, g)
$$

for any $f, g \in L_{\rho}$.

Other easy examples may be found in $[1,2]$.

Definition 2.3 Let $X_{w}$ be a modular metric space.

(1) The sequence $\left(x_{n}\right)_{n \in N}$ in $X_{w}$ is said to be $w$-convergent to $x \in X_{w}$ if and only if $w_{\lambda}\left(x_{n}, x\right) \rightarrow 0$ as $n \rightarrow \infty$ for some $\lambda>0$.

(2) The sequence $\left(x_{n}\right)_{n \in N}$ in $X_{w}$ is said to be $w$-Cauchy if $w_{\lambda}\left(x_{m}, x_{n}\right) \rightarrow 0$ as $m, n \rightarrow \infty$ for some $\lambda>0$.

(3) A subset $C$ of $X_{w}$ is said to be $w$-closed if the limit of a $w$-convergent sequence of $C$ always belongs to $C$.

(4) A subset $C$ of $X_{w}$ is said to be $w$-complete if any $w$-Cauchy sequence in $C$ is a convergent sequence and its limit is in $C$.

(5) A subset $C$ of $X_{w}$ is said to be $w$-bounded if for some $\lambda>0$, we have

$$
\delta_{w}(C)=\sup \left\{w_{\lambda}(x, y) ; x, y \in C\right\}<\infty .
$$

In general if $\lim _{n \rightarrow \infty} w_{\lambda}\left(x_{n}, x\right)=0$ for some $\lambda>0$, then we may not have $\lim _{n \rightarrow \infty} w_{\lambda}\left(x_{n}\right.$, $x)=0$ for all $\lambda>0$. Therefore, as it is done in modular function spaces, we say that $w$ satisfies $\Delta_{2}$-condition if this is the case, i.e., $\lim _{n \rightarrow \infty} w_{\lambda}\left(x_{n}, x\right)=0$ for some $\lambda>0$ implies $\lim _{n \rightarrow \infty} w_{\lambda}\left(x_{n}, x\right)=0$ for all $\lambda>0$. In [1] and [2], one can find a discussion about the connection between $w$-convergence and metric convergence with respect to the Luxemburg distances. In particular, we have

$$
\lim _{n \rightarrow \infty} d_{w}\left(x_{n}, x\right)=0 \quad \text { if and only if } \quad \lim _{n \rightarrow \infty} w_{\lambda}\left(x_{n}, x\right)=0 \quad \text { for all } \lambda>0,
$$

for any $\left\{x_{n}\right\} \in X_{w}$ and $x \in X_{w}$. And, in particular, we have $w$-convergence and $d_{w}$ convergence are equivalent if and only if the modular $w$ satisfies the $\Delta_{2}$-condition. Moreover, if the modular $w$ is convex, then we know that $d_{w}^{*}$ and $d_{w}$ are equivalent, which implies

$$
\lim _{n \rightarrow \infty} d_{w}^{*}\left(x_{n}, x\right)=0 \quad \text { if and only if } \quad \lim _{n \rightarrow \infty} w_{\lambda}\left(x_{n}, x\right)=0 \quad \text { for all } \lambda>0,
$$

for any $\left\{x_{n}\right\} \in X_{w}$ and $x \in X_{w}[1,2]$. Another question that arises in this setting is the uniqueness of $w$-limit. Assume that $w$ is regular, then we have the uniqueness of $w$-limit. Indeed, let $\left\{x_{n}\right\} \in X_{w}$ be a sequence such that $\left\{x_{n}\right\}$-converges to $a \in X_{w}$ and $b \in X_{w}$. Then we have

$$
w_{2}(a, b) \leq w_{1}\left(a, x_{n}\right)+w_{1}\left(x_{n}, b\right)
$$

for any $n \geq 1$. Our assumptions imply $w_{2}(a, b)=0$. Since $w$ is regular, we must have $a=b$ as claimed. 
Let us finish this section with the modular metric definitions of Lipschitzian mappings. The definitions are straightforward generalizations of their norm and metric equivalents [10].

Definition 2.4 Let $(X, w)$ be a modular metric space. Let $C$ be a nonempty subset of $X_{w}$. A mapping $T: C \rightarrow C$ is called a Lipschitzian mapping if there exists a constant $k \geq 0$ such that

$$
w_{1}(T(x), T(y)) \leq k w_{1}(x, y) \quad \text { for any } x, y \in C .
$$

The smallest such constant $k$ is known as $\operatorname{Lip}_{w}(T)$. A point $x \in C$ is called a fixed point of $T$ whenever $T(x)=x$. The set of fixed points of $T$ is denoted by Fix $(T)$.

In the next definition, we introduce the concept of pointwise contraction mappings in this setting.

Definition 2.5 Let $(X, w)$ be a modular metric space. Let $C$ be a nonempty subset of $X_{w}$. A mapping $T: C \rightarrow C$ is called

(i) a contraction mapping if there exists a constant $k \in[0,1)$ such that

$$
w_{1}(T(x), T(y)) \leq k w_{1}(x, y) \quad \text { for any } x, y \in C ;
$$

(ii) a pointwise contraction mapping if there exists a function $\alpha: C \rightarrow[0,1)$ such that

$$
w_{1}(T(x), T(y)) \leq \alpha(x) w_{1}(x, y) \quad \text { for any } x, y \in C .
$$

In $[1,2]$ the author defined Lipschitzian mappings in modular metric spaces and proved some fixed point theorems. Our definition is more general. Indeed, in the case of modular function spaces, it is proved in [10] that

$$
w_{\lambda}(T(x), T(y)) \leq w_{\lambda}(x, y) \text { for any } \lambda>0
$$

if and only if

$$
d_{w}(T(x), T(y)) \leq d_{w}(x, y) .
$$

Moreover, an example is given to show that

$$
w_{1}(T(x), T(y)) \leq w_{1}(x, y),
$$

but $T$ is not Lipschitzian with respect to $d_{w}$ with constant 1 .

\section{Banach contraction principle in modular metric spaces}

The statement of Banach contraction principle in modular metric spaces is as follows.

Theorem 3.1 Let $(X, w)$ be a modular metric space. Assume that $w$ is regular. Let $C$ be a nonempty subset of $X_{w}$. Assume that $C$ is $w$-complete and $w$-bounded, i.e., $\delta_{w}(C)=$ 
$\sup \left\{w_{1}(x, y) ; x, y \in C\right\}<\infty$. Let $T: C \rightarrow C$ be a contraction. Then $T$ has a unique fixed point $x_{0}$. Moreover, the orbit $\left\{T^{n}(x)\right\}$ w-converges to $x_{0}$ for each $x \in C$.

Proof Since $T$ is a contraction, therefore there exists $k \in[0,1)$ such that

$$
w_{1}(T(x), T(y)) \leq k w_{1}(x, y) \quad \text { for any } x, y \in C .
$$

First note that $T$ has at most one fixed point. Indeed, let $a, b \in C$ be two fixed points of $T$. Then we have

$$
w_{1}(a, b)=w_{1}(T(a), T(b)) \leq k w_{1}(a, b) .
$$

Our assumption on $C$ implies $w_{1}(a, b)<\infty$. This forces $w_{1}(a, b)=0$, which implies $a=b$ because $w$ is regular. Next we fix $x \in C$. Then we have

$$
w_{1}\left(T^{n+h}(x), T^{n}(x)\right) \leq k^{n} w\left(T^{h}(x), x\right) \leq k^{n} \delta_{w}(C)
$$

for any $n \geq 1$ and $h \geq 1$. This clearly implies that $\left\{T^{n}(x)\right\}$ is $w$-Cauchy. Since $C$ is $w$ complete, therefore $\left\{T^{n}(x)\right\} w$-converges to some point $x_{0} \in C$. Next let us show that $x_{0}$ is a fixed point of $T$. Indeed, we have

$$
w_{2}\left(x_{0}, T\left(x_{0}\right)\right) \leq w_{1}\left(x_{0}, T^{n}(x)\right)+w_{1}\left(T\left(x_{0}\right), T^{n}(x)\right),
$$

which implies

$$
w_{2}\left(x_{0}, T\left(x_{0}\right)\right) \leq w_{1}\left(x_{0}, T^{n}(x)\right)+k w_{1}\left(x_{0}, T^{n-1}(x)\right)
$$

for any $n \geq 1$. Since $\left\{T^{n}(x)\right\} w$-converges to $x_{0}$, therefore we get $w_{2}\left(x_{0}, T\left(x_{0}\right)\right)=0$, i.e., $T\left(x_{0}\right)=x_{0}$. Since $T$ has at most one fixed point, we conclude that any orbit of $T$ $w$-converges to the only fixed point $x_{0}$ of $T$.

Remark 3.1 In the classical Banach contraction principle, the metric space is not supposed to be bounded. In fact the contractive condition of the mapping implies that any orbit is bounded. In the case of a modular metric space, due to the failure of the triangle inequality, it is not true that the contractive condition of the mapping implies the boundedness of the orbit. Note that if $T$ has a fixed point, then it is obvious that an orbit of $T$ is bounded. Conversely, if $T$ has a bounded orbit $\left\{T^{n}(x)\right\}$ for some $x \in C$, then we have

$$
w_{1}\left(T^{n+h}(x), T^{n}(x)\right) \leq k^{n} w_{1}\left(T^{h}(x), x\right) \leq k^{n} \sup _{n, m \geq 1} w_{1}\left(T^{n}(x), T^{m}(x)\right)
$$

for any $n \geq 1$ and $h \geq 1$. This clearly implies that $\left\{T^{n}(x)\right\}$ is $w$-Cauchy. Since $C$ is $w$-complete, then $\left\{T^{n}(x)\right\}$ is $w$-convergent to some $x_{0} \in C$. Since

$$
w_{1}\left(T^{n}(x), T\left(x_{0}\right)\right) \leq k w_{1}\left(T^{n-1}(x), x_{0}\right), \quad n=1,2, \ldots
$$


we conclude that $\left\{T^{n}(x)\right\} w$-converges to $T\left(x_{0}\right)$. Since $w$ is regular, we must have $T\left(x_{0}\right)=x_{0}$, i.e., $x_{0}$ is the only fixed point of $T$. Next, for any $z \in C$ such that $w_{1}\left(z, x_{0}\right)<\infty$, we have

$$
w_{1}\left(T^{n}(z), x_{0}\right)=w_{1}\left(T^{n}(z), T^{n}\left(x_{0}\right)\right) \leq k^{n} w_{1}\left(z, x_{0}\right)
$$

for any $n \geq 1$. Hence the orbit $\left\{T^{n}(z)\right\} w$-converges to $x_{0}$ as well. In this remark, we show how one has to be careful when dealing with modulars. Indeed, a modular may take infinite value. This is the problem that the authors of [30] did not pay attention to. This was also pointed out in the short note [31]. In fact, the authors of [30] did try to fix this problem in another short note [32] but they used the triangle inequality in their proof knowing that $w$ does not in general satisfy the triangle inequality. Therefore our Theorem 3.1 properly establishes the classical Banach contraction principle in the best possible way in modular metric spaces and improves a number of earlier known results in this setting.

\section{Pointwise contraction mappings in modular metric spaces}

Pointwise contractive behavior was introduced in [22] to extend the contractive behavior in the Banach contraction principle. The central fixed point result in the metric setting for such mappings is the following theorem.

Theorem 4.1 $[22,23]$ Let $K$ be a weakly compact convex subset of a Banach space and suppose that $T: K \rightarrow K$ is a pointwise contraction. Then $T$ has a unique fixed point $x_{0}$. Moreover, the orbit $\left\{T^{n}(x)\right\}$ converges to $x_{0}$ for each $x \in K$.

The main focus of this paper is on the fixed point problem, so we would like to make some observations here. It is easy to prove that a pointwise contraction $T: C \rightarrow C$ has a fixed point in the same way as in the case of contractions. Indeed, let $a$ be a fixed point of $T$. Then the orbit $\left\{T^{n}(x)\right\} w$-converges to $a$ for each $x \in C$ such that $w_{1}(x, a)<\infty$. Indeed, we have

$$
w_{1}\left(a, T^{n}(x)\right)=w_{1}\left(T^{n}(a), T^{n}(x)\right) \leq \alpha(a)^{n} w_{1}(a, x)
$$

for any $x \in C$. Since $\alpha(a)<1$, we conclude that $\left\{T^{n}(x)\right\} w$-converges to $a$. Moreover, if $b$ is another fixed point of $T$, then from $w_{1}(a, b)<\infty$, we must have $a=b$. But it is not clear how to prove the existence of the fixed point from the convergence of the orbits, which is the case in the classical proof of the Banach contraction principle. In this case, we have to use a different technique than the one used in Theorem 3.1.

Let $(X, w)$ be a modular metric space. In the rest of this work, we assume that $w$ satisfies the Fatou property, i.e., if $\left\{x_{n}\right\} w$-converges to $x$ and $\left\{y_{n}\right\} w$-converges to $y$, then we must have

$$
w_{1}(x, y) \leq \liminf _{n \rightarrow \infty} w_{1}\left(x_{n}, y_{n}\right)
$$

For any $x \in X_{w}$ and $r \geq 0$, we define the modular ball

$$
B_{w}(x, r)=\left\{y \in X_{w} ; w_{1}(x, y) \leq r\right\} .
$$


Note that if $w$ satisfies the Fatou property, then modular balls are $w$-closed. An admissible subset of $X_{w}$ is defined as an intersection of modular balls. Denote by $\mathcal{A}_{w}\left(X_{w}\right)$ the family of admissible subsets of $X_{w}$. Note that $\mathcal{A}_{w}\left(X_{w}\right)$ is stable under intersection. At this point we introduce some notation which will be used in the remainder of this work. For a subset $A$ of a modular metric space $X_{w}$, set

$$
\operatorname{cov}_{w}(A)=\cap\{B: B \text { is a modular ball and } A \subset B\} .
$$

Recall that $A$ is $w$-bounded if $\delta_{w}(A)=\sup \left\{w_{1}(x, y) ; x, y \in A\right\}<\infty$.

Definition 4.1 Let $(X, w)$ be a modular metric space. We say that $\mathcal{A}_{w}\left(X_{w}\right)$ is compact if any family $\left(A_{\alpha}\right)_{\alpha \in \Gamma}$ of elements of $\mathcal{A}_{w}\left(X_{w}\right)$ has a nonempty intersection provided $\bigcap_{\alpha \in F} A_{\alpha} \neq \emptyset$ for any finite subset $F \subset \Gamma$.

Remark 4.1 Note that if $\mathcal{A}_{w}\left(X_{w}\right)$ is compact, then $X_{w}$ is $w$-complete. Indeed, let $\left\{x_{n}\right\} \subset X_{w}$ be a $w$-Cauchy sequence. Set

$$
r_{n}=\sup _{m, s \geq n} w_{1}\left(x_{m}, x_{s}\right)
$$

for any $n \geq 1$. Since $\left\{x_{n}\right\}$ is a $w$-Cauchy sequence, then $\lim _{n \rightarrow \infty} r_{n}=0$. By the definition of $r_{n}$, we get $x_{m} \in B_{w}\left(x_{n}, r_{n}\right)$ for any $n \geq 1$ and $m \geq n$. Hence, for any $n_{1}, n_{2}, \ldots, n_{p} \geq 1$, we have

$$
x_{m} \in \bigcap_{1 \leq i \leq p} B_{w}\left(x_{n_{i}}, r_{n_{i}}\right)
$$

for any $m \geq \max \left\{n_{1}, n_{2}, \ldots, n_{p}\right\}$. Since $\mathcal{A}_{w}\left(X_{w}\right)$ is compact, then

$$
\Omega=\bigcap_{n \geq 1} B_{w}\left(x_{n}, r_{n}\right) \neq \emptyset .
$$

If $z \in \Omega$, then we have $w_{1}\left(x_{n}, z\right) \leq r_{n}$ for any $n \geq 1$. Hence $\left\{x_{n}\right\} w$-converges to $z$, which completes the proof of our statement.

The main result in $[22,23]$ may be stated in modular metric spaces as follows.

Theorem 4.2 Let $(X, w)$ be a modular metric space. Let $C$ be a nonempty $w$-closed wbounded subset of $X_{w}$. Assume that the family $\mathcal{A}_{w}(C)$ is compact. Let $T: C \rightarrow C$ be a pointwise contraction. Then $T$ has a unique fixed point $x_{0} \in C$. Moreover, the orbit $\left\{T^{n}(x)\right\}$ $w$-converges to $x_{0}$ for each $x \in C$.

Proof Since $\mathcal{A}_{w}(C)$ is compact, therefore there exists a minimal nonempty $K \in \mathcal{A}_{w}(C)$ such that $T(K) \subset K$. It is easy to check that $\operatorname{cov}_{w}(T(K))=K$. Let us prove that $\delta_{w}(K)=0$, i.e., $K$ is reduced to one point. Indeed, since $C$ is $w$-bounded, then $\delta_{w}(K)<\infty$, i.e., $K$ is also $w$-bounded. As $T$ is a pointwise contraction, so there exists a mapping $\alpha: C \rightarrow[0,1)$ such that

$$
w_{1}(T(x), T(y)) \leq \alpha(x) w_{1}(x, y) \quad \text { for any } x, y \in C .
$$


For any $x \in K$, set $r_{x}(K)=\sup \left\{w_{1}(x, y) ; y \in K\right\}$. We have $r_{x}(K) \leq \delta_{w}(K)$ for any $x \in K$. Moreover, it is easy to check that $T(K) \subset B_{w}\left(T(x), \alpha(x) r_{x}(K)\right)$ for any $x \in K$, which implies $\operatorname{cov}_{w}(T(K)) \subset B_{w}\left(T(x), \alpha(x) r_{x}(K)\right)$. So, $r_{T(x)}(K) \leq \alpha(x) r_{x}(K)$ for any $x \in K$. Next we fix $a \in K$ and define

$$
K_{a}=\left\{x \in K ; r_{x}(K) \leq r_{a}(K)\right\}
$$

Clearly, $K_{a}$ is not empty since $a \in K_{a}$. Moreover, we have

$$
K_{a}=\bigcap_{x \in K} B_{w}\left(x, r_{a}(K)\right) \cap K \in \mathcal{A}_{w}(C) .
$$

And since $r_{T(x)}(K) \leq \alpha(x) r_{x}(K)$ for any $x \in K$, we get $T\left(K_{a}\right) \subset K_{a}$. The minimality behavior of $K$ implies $K_{a}=K$. In particular, we have $r_{x}(K)=r_{a}(K)$ for any $x \in K$. Hence $\delta_{w}(K)=$ $\sup _{x \in K} r_{x}(K)=r_{a}(K)$ for any $a \in K$. Hence $\delta_{w}(K) \leq \alpha(a) \delta_{w}(K)$. And since $\alpha(a)<1$, we get $\delta_{w}(K)=0$, i.e., $K$ is reduced to one point which is fixed by $T$. Hence the fixed point set of $T$ is not empty. The remaining conclusion of the theorem follows from the general properties of pointwise contractions.

\section{Competing interests}

The authors declare that they have no competing interests.

\section{Authors' contributions}

All authors participated in the design of this work and performed equally. All authors read and approved the final manuscript.

\section{Author details}

${ }^{1}$ Department of Mathematics, King Abdulaziz University, P.O. Box 80203, Jeddah, 21589, Saudi Arabia. ${ }^{2}$ Department of Mathematical Science, The University of Texas at El Paso, El Paso, 79968, USA. ${ }^{3}$ Department of Mathematics and Statistics, King Fahd University of Petroleum \& Minerals, Dhahran, 31261, Saudi Arabia.

Received: 28 March 2013 Accepted: 3 June 2013 Published: 25 June 2013

\section{References}

1. Chistyakov, W: Modular metric spaces, I: basic concepts. Nonlinear Anal. 72(1), 1-14 (2010)

2. Chistyakov, W: Modular metric spaces, II: application to superposition operators. Nonlinear Anal. 72(1), 15-30 (2010)

3. Nakano, H: Modulared Semi-Ordered Linear Spaces. Maruzen, Tokyo (1950)

4. Musielak, J: Orlicz Spaces and Modular Spaces. Lecture Notes in Math., vol. 1034. Springer, Berlin (1983)

5. Orlicz, W: Collected Papers. Part I, II. Panstwowe Wydawnictwo Naukove, Warsaw (1988)

6. Diening, L: Theoretical and numerical results for electrorheological fluids. Ph.D. thesis, University of Freiburg, Germany (2002)

7. Ruzicka, M: Electrorheological Fluids Modeling and Mathematical Theory. Springer, Berlin (2002)

8. Harjulehto, P, Hästö, P, Koskenoja, M, Varonen, S: The Dirichlet energy integral and variable exponent Sobolev spaces with zero boundary values. Potential Anal. 25(3), 205-222 (2006)

9. Heinonen, J, Kilpeläinen, T, Martio, O: Nonlinear Potential Theory of Degenerate Elliptic Equations. Oxford University Press, Oxford (1993)

10. Khamsi, MA, Kozlowski, WK, Reich, S: Fixed point theory in modular function spaces. Nonlinear Anal. 14, 935-953 (1990)

11. Khamsi, MA, Kozlowski, WK: On asymptotic pointwise contractions in modular function spaces. Nonlinear Anal. 73 2957-2967 (2010)

12. Khamsi, MA, Kozlowski, WK: On asymptotic pointwise nonexpansive mappings in modular function spaces. J. Math. Anal. Appl. 380, 697-708 (2011)

13. Belluce, LP, Kirk, WA: Fixed-point theorems for families of contraction mappings. Pac. J. Math. 18, $213-217$ (1966)

14. Belluce, LP, Kirk, WA: Nonexpansive mappings and fixed-points in Banach spaces. III. J. Math. 11, 474-479 (1967)

15. Browder, FE: Nonexpansive nonlinear operators in a Banach space. Proc. Natl. Acad. Sci. USA 54, 1041-1044 (1965)

16. Bruck, RE: A common fixed point theorem for a commuting family of nonexpansive mappings. Pac. J. Math. 53, 59-71 (1974)

17. DeMarr, RE: Common fixed-points for commuting contraction mappings. Pac. J. Math. 13, 1139-1141 (1963)

18. Lim, TC: A fixed point theorem for families of nonexpansive mappings. Pac. J. Math. 53, 487-493 (1974)

19. Goebel, K, Reich, S: Uniform Convexity, Hyperbolic Geometry, and Nonexpansive Mappings. Series of Monographs and Textbooks in Pure and Applied Mathematics, vol. 83. Dekker, New York (1984) 
20. Goebel, K, Sekowski, T, Stachura, A: Uniform convexity of the hyperbolic metric and fixed points of holomorphic mappings in the Hilbert ball. Nonlinear Anal. TMA 4, 1011-1021 (1980)

21. Kirk, WA: A fixed point theorem in CAT(0) spaces and $\mathbb{R}$-trees. Fixed Point Theory Appl. 2004(4), 309-316 (2004)

22. Kirk, WA: Fixed points of asymptotic contractions. J. Math. Anal. Appl. 277, 645-650 (2003)

23. Kirk, WA: Asymptotic pointwise contractions. In: Plenary Lecture, the 8th International Conference on Fixed Point Theory and Its Applications, Chiang Mai University, Thailand, July 16-22 2007 (2007)

24. Hussain, N, Khamsi, MA: On asymptotic pointwise contractions in metric spaces. Nonlinear Anal. 71(10), 4423-4429 (2009)

25. Kirk, WA, Hong-Kun, Xu: Asymptotic pointwise contractions. Nonlinear Anal. 69, 4706-4712 (2008)

26. Khamsi, MA, Kirk, WA: An Introduction to Metric Spaces and Fixed Point Theory. Wiley, New York (2001)

27. Kozlowski, WM: Modular Function Spaces. Series of Monographs and Textbooks in Pure and Applied Mathematics, vol. 122. Dekker, New York/Basel (1988)

28. Kozlowski, WM: Notes on modular function spaces I. Comment. Math. 28, 91-104 (1988)

29. Kozlowski, WM: Notes on modular function spaces II. Comment. Math. 28, 105-120 (1988)

30. Mongkolkeha, C, Sintunavarat, W, Kumam, P: Fixed point theorems for contraction mappings in modular metric spaces. Fixed Point Theory Appl. (2011). doi:10.1186/1687-1812-2011-93

31. Dehghan, $\mathrm{H}$, Eshaghi Gordji, M, Ebadian, A: Comment on 'Fixed point theorems for contraction mappings in modular metric spaces, Fixed Point Theory and Applications, doi:10.1186/1687-1812-2011-93, 20 pages', Fixed Point Theory Appl. (2012). doi:10.1186/1687-1812-2012-144

32. Mongkolkeha, C, Sintunavarat, W, Kumam, P: Correction: Fixed point theorems for contraction mappings in modular metric spaces, Fixed Point Theory Appl. 2011, 2011:93. Fixed Point Theory Appl. 2012, Article ID 103 (2012). doi:10.1186/1687-1812-2012-103

doi:10.1186/1687-1812-2013-163

Cite this article as: Abdou and Khamsi: Fixed point results of pointwise contractions in modular metric spaces. Fixed Point Theory and Applications 2013 2013:163.

\section{Submit your manuscript to a SpringerOpen ${ }^{\circ}$ journal and benefit from:}

- Convenient online submission

- Rigorous peer review

- Immediate publication on acceptance

Open access: articles freely available online

- High visibility within the field

- Retaining the copyright to your article 